\title{
Compromiso laboral del trabajo social en el sector salud
}

Javier Carreón Guillén

Universidad Nacional Autónoma de México, Escuela Nacional de Trabajo Social javierg@unam.mx

\section{Resumen}

En virtud de que el trabajo social está inmerso en políticas públicas y programas sociales que van dirigidos a grupos vulnerables, marginados o excluidos, es menester analizar su compromiso considerando su relación con la gestión para el tratamiento de enfermedades y la rehabilitación en instituciones del sector salud. En tal sentido, el objetivo del presente estudio fue establecer la confiabilidad y validez de un instrumento que mide el compromiso laboral en el sector salud. Para tal propósito, se utilizó una muestra no probabilística de 125 profesionales del trabajo social con experiencia en implementación de programas sociales y seguimiento de estrategias institucionales para la promoción de la salud. Se establecieron la normalidad, confiabilidad y validez de la escala que midió el compromiso laboral; además de un modelo estructural reflejante en el que el compromiso hacia la institución se relacionó positivamente con el compromiso laboral. Los índices de ajuste y residual corroboraron la hipótesis de multidimensionalidad del compromiso laboral.

Palabras claves: políticas públicas, sector salud, trabajo social, compromiso laboral 


\title{
Commitment to work in the health sector social work
}

\begin{abstract}
Given that social work is involved in public policy and social programs that target vulnerable, marginalized or excluded groups, it is necessary to analyze its commitment considering its relation to the management for the treatment of illness and rehabilitation of health sector institutions. In this regard, the objective of this study was to establish the reliability and validity of an instrument for measuring work commitment in the health sector. For this purpose, we used a nonrandom sample of 125 social work professionals with experience in implementing social programs and monitoring of institutional strategies for health promotion. Normality, reliability and validity on the scale that measured labor commitment were established. A reflective structural model was established in which commitment to the institution was positively related to work engagement. The residual and fit indices corroborated the multidimensionality hypothesis of work commitment.
\end{abstract}

Keywords: public policy, health sector, social work, occupational commitment

\section{Introducción}

En México, las políticas y programas sociales suponen la asistencia a grupos vulnerables, marginados o excluidos a través de la práctica profesional del trabajo social. En el área de la salud, la gestión y promoción son ámbitos de intervención del trabajo social; es decir, a medida que el Estado incentiva el desarrollo humano en sus esferas de salud, educación y empleo incide en las estrategias institucionales de prevención y tratamiento de enfermedades. Sin embargo, la maquinaria político-institucional del sector salud requiere de talentos comprometidos con grupos sociales de escasos recursos, ya que entre éstos se formarán las redes para el desarrollo local (Barranco, Delgado, Melin y Quintana, 2010). Por ello, resulta relevante estudiar los indicadores de compromiso laboral en profesionistas vinculados con los programas de asistencia social. Un diagnóstico de las áreas de oportunidad y las virtudes del sistema de gestión y promoción de la salud permitirá discutir la emergencia de nuevos paradigmas de asistencia social ${ }^{1}$ centrados en los promotores de desarrollo humano (Melano, 2007).

\footnotetext{
${ }^{1}$ Al aludir a la asistencia social, no se está pensando, de momento, en el término peyorativo de asistencialismo. En la parte empírica de este trabajo se podrá apreciar una interesante problemática al respecto; no obstante, la nueva tecnología lo denomina servicios sociales.
} 
No obstante que las instituciones públicas están circunscritas a un Plan de Desarrollo Nacional, el ejercicio profesional del trabajo social a menudo está condicionado por situaciones locales, institucionales, o bien por el clima laboral, el salario o el estrés que implica la atención personalizada (Ocampo, 2008). En este sentido, es menester establecer las dimensiones del compromiso organizacional de los profesionales del trabajo social, ya que un alto grado de compromiso sugiere un nivel de atención eficiente que podría traducirse en una evaluación favorable de las políticas sociales y los programas institucionales.

En el caso del sector salud, el compromiso laboral - al estar asociado con la gestión y promoción de la salud - está configurado por indicadores que se encargan de enaltecer las estructuras y políticas institucionales para predecir acciones personales (Ruiz, 2010). Así, el compromiso laboral ha sido un transmisor de las relaciones y conflictos al interior de una institución que inhibe o potencializa la satisfacción laboral. A medida que el compromiso profesional transfiere valores organizacionales de interrelación colaborativa, incide asimismo en las expectativas de los miembros de una organización (Santarsierro, 2012). Tal proceso supone la coexistencia entre indicadores del compromiso laboral que por su naturaleza son complementarios y opuestos; es decir, el compromiso organizacional contiene dos procesos de socialización: de primer orden alusivo a principios que ubican al individuo en un contexto y de segundo orden relativo a principios que lo identifican en una estructura de relaciones de poder; ambas pueden ser complementarias u opuestas.

En el ámbito del trabajo social, las instituciones fungen como estructuras de socialización de segundo orden para incidir en las percepciones, creencias, actitudes, decisiones y acciones de sus empleados. En principio, las instituciones son un escenario de racionalidad política en la que el Estado esgrime programas de asistencia social que los profesionales del trabajo social deben seguir y, en todo caso, perfeccionar dicho sistema para lograr la evaluación favorable de las políticas públicas y los programas sociales; cabe señalar que dicho proceso de racionalidad política puede ser complementario o antagónico a los principios que guían el compromiso personal, interpersonal, familiar o colaborativo en los empleados de una institución pública (Pastor, 2012). Por ello, es preciso esclarecer las dimensiones del compromiso organizacional a partir de la complejidad que implica el ejercicio profesional del trabajo social. 
En ese sentido, este trabajo busca establecer las dimensiones del compromiso laboral considerando niveles de complejidad institucional que enmarcarían el desencuentro con indicadores de un compromiso derivado de la socialización primaria. Ante tales elementos surge una interrogante que, de una manera u otra, vendrá a guiar las diversas reflexiones que se plantean: ¿Cuáles son las dimensiones del compromiso laboral en profesionales del trabajo social que laboran en instituciones públicas, pero han tenido una formación crítica en torno al ejercicio de su profesión y han sido socializados bajo principios colectivistas en donde los intereses de la mayoría prevalecen sobre los objetivos personales o institucionales?

La respuesta a tal cuestión está confinada a los estudios psicológicos organizacionales relativos al compromiso laboral. Se trata de cuatro dimensiones en las que el compromiso está indicado por el apoyo, el reconocimiento, el aprendizaje y la valoración (véase figura 1); en otras palabras, el compromiso laboral puede ser observado a partir del apoyo que la organización le otorga al individuo para su desarrollo personal, familiar y laboral (hipótesis 1). Así, los líderes de la organización que reconocen acciones específicas al individuo incentivan el reconocimiento de los grupos laborales, familiares o interpersonales en los que está inserto el individuo (hipótesis 2). De este modo, una acción concreta que implica un reconocimiento puntual supone un aprendizaje específico como síntoma de compromiso laboral (hipótesis 3). Por último, las organizaciones con compromiso social y que transfieren estos valores a los individuos generan una serie de valores personales que pueden ser observados como síntomas de su compromiso laboral (hipótesis 4). 
Figura 1

Modelo de relaciones especificadas

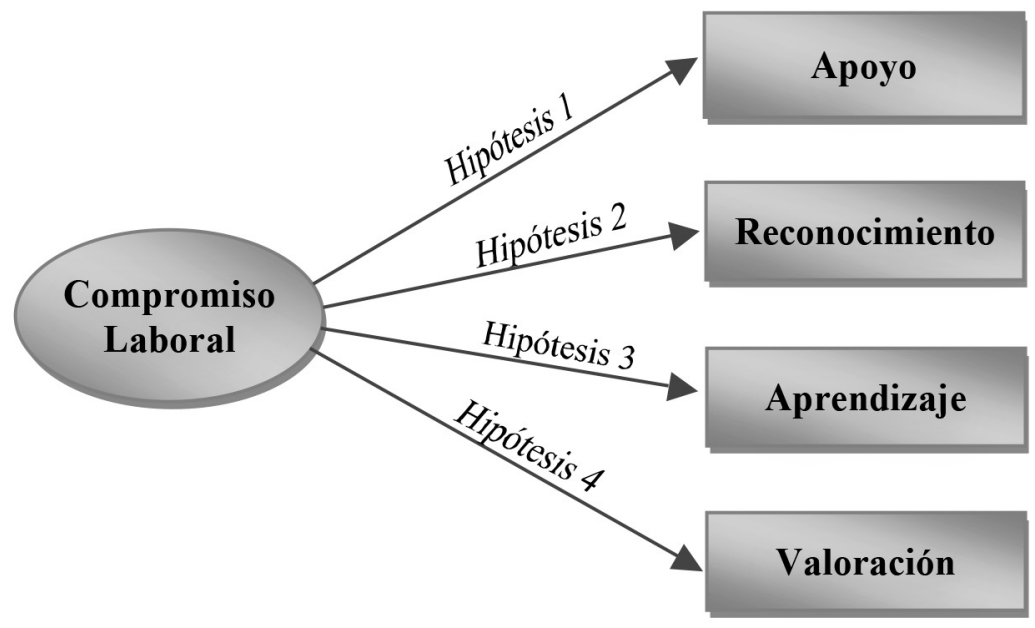

\section{Teoría del compromiso laboral}

El compromiso laboral está imbricado en un sistema psicológico organizacional que parte del clima y termina en la satisfacción (véase figura 2). En tal sistema, el compromiso se interrelaciona con el liderazgo y el desempeño para mediar la relación entre clima y satisfacción. Se trata de supuestos según los cuales indican que un incremento en las relaciones de tareas y humanas impacta en el liderazgo, el compromiso y el desempeño, lo que a su vez incide en un aumento en la satisfacción de vida; es decir, el capital humano en las organizaciones parece estar inmerso en una serie de actividades y personalidades que la llevan a asumir roles de liderazgo y dimensiones de compromiso que incrementan su desempeño. Aunque tal proceso está, más bien, orientado a una mayor satisfacción personal, la organización parece interceder en tales propósitos (Castro, 2006; Castro y Martins, 2009; Salgado, Remeseiro e Iglesias, 1996; Tejero y Fernández, 2009; Vecina, Chacón y Sueiro, 2009; Yáñez, Arenas y Ripoll, 2010). 
Figura 2

Sistemas psicológicos organizacionales

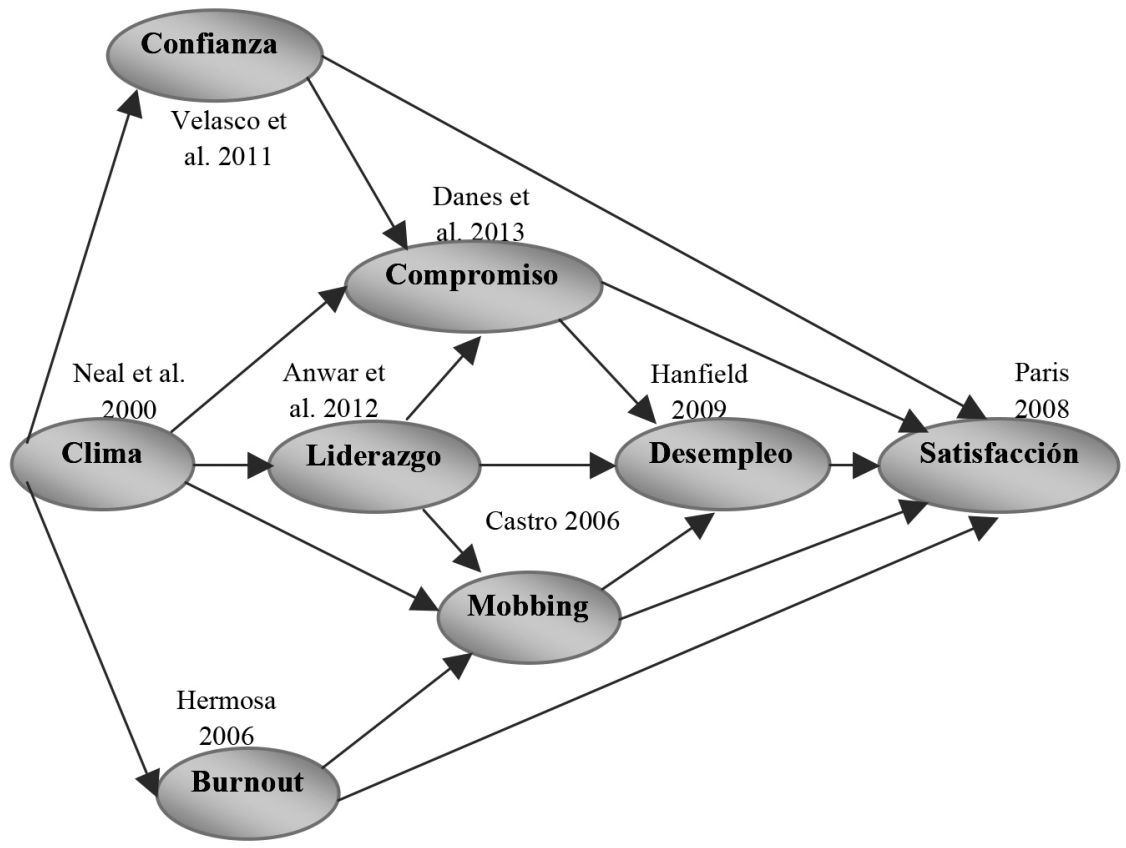

Fuente: García-Lirios (2011).

La psicología de las organizaciones ha planteado que los procesos productivos son inherentes a los procesos cognitivos de quienes laboran en una organización (Ríos, Téllez y Ferrer, 2010).

En referencia con el desarrollo organizacional, los estudios psicológicos organizacionales han demostrado que el compromiso es un factor de sistematización de la producción. En tal sentido, el compromiso es asumido como un conjunto de acciones, roles, motivos y expectativas que generan una dinámica colaborativa entre los integrantes de un grupo de trabajo u organización productiva.

El modelo de compromiso organizacional de Caykoylu, Egri, Havlovic y Bradley (2011) plantea siete causas relativas al empoderamiento, motivación, identidad, confianza, ambigüedad y conflicto, las cuales inciden sobre el compromiso a través de la satisfacción (véase figura 3). 
Figura 3

Modelo de compromiso organizacional

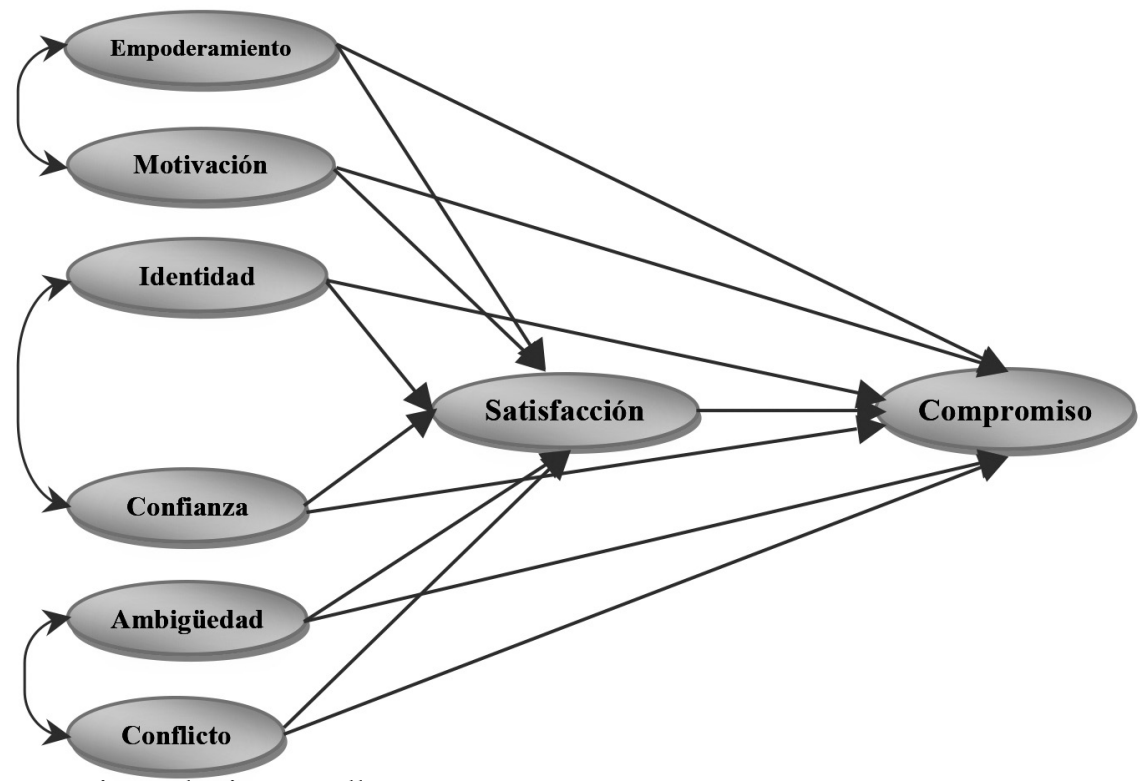

Fuente: Caykoylu, Egri, Havlovic y Bradley (2011).

Un aumento en el empoderamiento y la motivación propiciaría un incremento en la satisfacción y el compromiso. Sin embargo, la reducción de ambigüedad y conflicto al relacionarse negativamente con la satisfacción inciden en un incremento del compromiso. Antes bien, la identidad y confianza al aumentar también influyen en la satisfacción y el compromiso (Hermosa, 2006).

Si el compromiso organizacional es determinado por el empoderamiento, motivación, identidad, confianza, ambigüedad y conflicto a través de la satisfacción, entonces el compromiso puede ser definido como el resultado de la interrelación entre factores organizacionales de orden humano en referencia a la relación entre líderes y empleados. En este sentido, el compromiso está en función de los deseos personales y las visiones organizacionales; se trata de un indicador de equidad y justicia en el que los líderes se relacionan con empleados a partir de un equilibrio entre libertades, capacidades y responsabilidades. 
A partir de tales presupuestos, los psicólogos organizacionales han asumido que el compromiso es un producto más que un proceso permanente de identidad. Quienes asumen un compromiso con sus empresas son considerados como un producto de la dinámica organizacional más que como individuos con personalidades y valores colaborativos. En tal sentido, el reclutamiento y la selección de prospectos no se realiza con base en sus características individuales, sino en sus capacidades y estrategias de afrontamiento ante la emergencia de los conflictos, el riesgo y la incertidumbre (Moreno, Ríos, Canto, García y Perles, 2010).

El compromiso organizacional abre la discusión en torno a la relación entre organización e individuo. La influencia de la primera en el segundo parece ser corroborada con los estudios psicológicos organizacionales, pero el compromiso - en tanto sistematización de funciones y resultados - va más allá del individuo y la organización. El compromiso laboral alude a un conjunto de principios morales y valorativos característicos de los líderes que, en su afán por conseguir los objetivos trazados, creen firmemente en los ideales de la productividad, el orden y la sistematización de las funciones organizacionales (Figeiredo, Grau, Gil y García, 2012).

En síntesis, el compromiso es un conjunto de creencias, actitudes y acciones que reducen la incertidumbre e incrementan la propensión al futuro. El aumento de expectativas de riesgo disminuiría la motivación para el trabajo y desordenaría al sistema de relaciones humanas afectando al desempeño de cada miembro.

\section{Estudios psicológicos del compromiso laboral}

Los estudios psicológicos organizacionales han establecido dimensiones de compromiso laboral a partir de su relación con variables de orden organizacional (véase cuadro 1). Principalmente, el compromiso laboral ha correlacionado con la cultura laboral (Wallece, Hunt y Richards, 1999), el clima de tareas (Neal, Griffin y Hart, 2000), la satisfacción de vida (Mañas, Boada, González y Agullo, 2007), el reconocimiento social (Mendoza, Orgambídez y Carrasco, 2010), el liderazgo transformacional (Anwar y Norulkamar, 2012), el desempeño productivo (Tayo y Adeyemi, 2012) y el espíritu emprendedor (Danes y Joyoung, 2013). 


\section{Cuadro 1 \\ Estado del arte}

\begin{tabular}{|c|c|c|}
\hline Año & Autor & Hallazgos \\
\hline 1999 & $\begin{array}{l}\text { Wallece, Hunt y } \\
\text { Richards }\end{array}$ & $\begin{array}{l}\text { El clima de conflictos y tareas correlacionaron con la cultura organizacional ( } \mathrm{r} \text { respectivas } \\
\text { de } .31 \text { y } .34 \text { con significancia menor a } 0.010 \text { ). Los climas de conflicto correlacionaron } \\
\text { negativamente con los valores organizacionales: eficiencia, efectividad, elitismo, apertura, } \\
\text { justicia, oportunidad, poder y endogrupo. El clima de cooperación correlacionó positivamente } \\
\text { con cada uno de los valores. }\end{array}$ \\
\hline 2000 & $\begin{array}{l}\text { Neal, Griffin y } \\
\text { Hart }\end{array}$ & $\begin{array}{l}\text { El clima organizacional influyó en el clima de seguridad ( } \beta=0.54) \text {, el clima de seguridad } \\
\text { sobre el conocimiento de seguridad }(\beta=0.58) \text {, el conocimiento de seguridad sobre los } \\
\text { procedimientos de seguridad }(\beta=0.35) \text { y sobre la participación de seguridad }(\beta=0.28) \text {, el } \\
\text { clima de seguridad sobre la motivación de seguridad }(\beta=0.43) \text { y sobre la participación de } \\
\text { seguridad }(\beta=0.23) \text {, la motivación de seguridad sobre los procedimientos de seguridad }(\beta= \\
.57) \text { y sobre la participación de seguridad }(\beta=0.29) \text {. }\end{array}$ \\
\hline 2007 & $\begin{array}{l}\text { Mañas, Boada, } \\
\text { González y } \\
\text { Agullo }\end{array}$ & El sexo, la satisfacción laboral y de vida son predictores del compromiso organizacional. \\
\hline 2010 & $\begin{array}{l}\text { Mendoza, } \\
\text { Orgambídez y } \\
\text { Carrasco }\end{array}$ & $\begin{array}{l}\text { El factor de compromiso correlacionó con la orientación al cliente, sugerencias, } \\
\text { reconocimiento y formación }(\mathrm{r}=0.29, \mathrm{r}=0.29 ; \mathrm{r}=0.34 \mathrm{y} \mathrm{r}=0.23 \text {, todos con un nivel de } \\
\text { significancia menor a 0.05). }\end{array}$ \\
\hline 2012 & $\begin{array}{l}\text { Anwar } \\
\text { y Norulkamar }\end{array}$ & $\begin{array}{l}\text { El compromiso laboral se relacionó positivamente con el liderazgo, el desempeño y la } \\
\text { satisfacción ( } \mathrm{r} \text { respectiva de } 0.105,0.433,0.431 \text { y } 0.281 ; \mathrm{p}=0.05) \text {. El compromiso determinó } \\
\text { el desempeño laboral }(\beta=0.105 ; \mathrm{p}=0.05) \text { y la satisfacción }(\beta=0.43 ; \mathrm{p}=0.05) \text {. El liderazgo } \\
\text { transformacional y transaccional con el desempeño laboral }(\beta 0.152 \text { y } \beta=0.107 ; \mathrm{p}=0.05) \text {, } \\
\text { la satisfacción }(\beta=0.603 \text { y } \beta=0.305 ; \mathrm{p}=0.001) \text { y al compromiso }(\beta=0.431 \text { y } \beta=0.281 \text {; } \\
\mathrm{p}=0.001) \text {. }\end{array}$ \\
\hline 2012 & $\begin{array}{l}\text { Tayo } \\
\text { y Adeyemi }\end{array}$ & $\begin{array}{l}\text { Correlacionaron al compromiso organizacional con el desempeño laboral } \\
(\mathrm{r}=0.75 ; \mathrm{p}=0.05)\end{array}$ \\
\hline 2013 & $\begin{array}{l}\text { Danes } \\
\text { \& Joyoung }\end{array}$ & $\begin{array}{l}\text { La evaluación del compromiso empresarial fue determinada por la autoevaluación de } \\
\text { compromiso }(\beta=0.62) \text {. A su vez, se estableció una correlación con la autoevaluación del } \\
\text { compromiso empresarial }(\beta=0.17) \text {. Es decir, a medida que el grado de responsabilidad se } \\
\text { incrementa, el espíritu emprendedor también tiene un aumento en sus valores relativos al } \\
\text { compromiso y la autoevaluación. }\end{array}$ \\
\hline
\end{tabular}

Los estudios psicológicos del compromiso laboral han establecido relaciones causales entre esta variable y los estilos de liderazgo (Anwar y Norulkamar, 2012). A medida que el tipo de liderazgo se intensifica, se explica el incremento del compromiso laboral; es decir, los diferentes tipos de liderazgo repercuten en el aumento o disminución de las percepciones, actitudes, decisiones y acciones de los empleados. De la misma manera, la historia de desempeño repercute en el compromiso de incrementar la productividad en el futuro (Tayo y Adeyemi, 2012); dicho de otra forma, el compromiso laboral, las estructuras de mando, las relaciones de tarea, los conflictos y el estrés o la satisfacción parecen explicar el aumento o la disminución del compromiso que asumen los empleados en su estancia laboral, aunque 
para Mañas et al. (2007) el sexo y para Mendoza et al. (2010) la atención al cliente explican el grado de compromiso laboral.

Mañas et al. (2007), así como Anwar y Norulkamar (2012) coinciden en relacionar la satisfacción de vida con el compromiso. Tales hallazgos suponen una continuidad entre la socialización de primer y segundo orden; ya sea en un sentido colectivista o individualista, favorable o desfavorable, los principios que guían al individuo en un grupo familiar serían los mismos que los guían en un grupo laboral o productivo. A la luz de estos resultados, el compromiso laboral sería el último eslabón, al menos en el ámbito laboral, de una cadena de percepciones, creencias, actitudes, decisiones y acciones dirigidas desde el grupo primario en el que el individuo aprendió los símbolos básicos y en cuyo desarrollo nunca tuvo la oportunidad de cuestionar tales principios que ahora parecen guiarlo en su compromiso, productividad y satisfacción laboral. El estado del arte parece mostrar evidencias sólidas en torno a la complementariedad entre la socialización de primer orden y la socialización de segundo orden.

\section{Abordaje metodológico}

En la presente investigación se llevó a cabo un estudio correlacional. La selección de la muestra fue no probabilística de 125 profesionales ( 75 mujeres y 50 hombres con un promedio de edad de 33 años y 7 años de haber egresado) del trabajo social de centros de salud del estado de Morelos (México) con ingreso promedio mensual de 870 USD y siete años promedio de experiencia laboral . Considerando que el compromiso organizacional está influido por la política social y el programa de asistencia, se consideró conveniente como criterio de inclusión el conocer el Plan de Desarrollo Nacional e Institucional, así como las áreas de práctica profesional.

En el instrumento de recopilación de información se incluyeron preguntas relativas a sexo, edad, ingreso, procedencia, experiencia y situación civil como familiar. Se utilizó la Escala de Compromiso Laboral de García (2011), la cual incluye reactivos en torno a las percepciones de apoyo (4 ítems), reconocimiento (12 ítems), aprendizaje (11 ítems) y valoración laboral (8 ítems). Cada reactivo incluye cinco opciones de respuesta que van desde "nunca" hasta "siempre" (véase cuadro 2). 


\section{Cuadro 2}

\section{Operacionalización de factores}

\begin{tabular}{|c|c|c|c|c|c|}
\hline Variable & Definición & Ítem ejemplo & Ponderación & Medición & Ítems \\
\hline 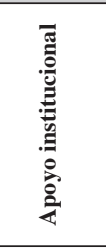 & $\begin{array}{l}\text { Se refiere a la percepción de } \\
\text { las facilidades otorgadas por } \\
\text { los líderes de la institución } \\
\text { para llevar a cabo la práctica } \\
\text { profesional en referencia a la } \\
\text { política social y los programas } \\
\text { institucionales. } \\
\end{array}$ & $\begin{array}{l}\text { "El centro de } \\
\text { salud cuenta con } \\
\text { los recursos que } \\
\text { la política social } \\
\text { necesita para ayudar } \\
\text { a la gente" }\end{array}$ & $\begin{array}{l}\text { Medición de } \\
\text { percepciones a partir de } \\
\text { frecuencias que van de } \\
\text { "nunca" hasta "siempre" }\end{array}$ & Ordinal & $\begin{array}{l}\stackrel{2}{\sigma} \\
\stackrel{2}{\sim} \\
\sim\end{array}$ \\
\hline 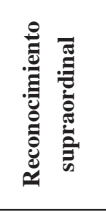 & $\begin{array}{l}\text { El conjunto de percepciones que } \\
\text { los empleados tienen respecto a } \\
\text { los logros que son enunciados } \\
\text { por sus líderes como acciones } \\
\text { extraordinarias. }\end{array}$ & $\begin{array}{l}\text { "Nuestro líder es } \\
\text { muy dado a elogiar } \\
\text { a quienes visitan } \\
\text { comunidades } \\
\text { lejanas" }\end{array}$ & $\begin{array}{l}\text { Medición de } \\
\text { percepciones a partir de } \\
\text { frecuencias que van de } \\
\text { "nunca" hasta "siempre" }\end{array}$ & Ordinal & $\begin{array}{l}\cong \\
\cong \\
= \\
0 \\
0 \\
\infty \\
0\end{array}$ \\
\hline 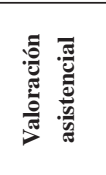 & $\begin{array}{l}\text { Son percepciones relativas } \\
\text { a la relevancia de la práctica } \\
\text { profesional que se lleva a cabo } \\
\text { considerando la supervisión de } \\
\text { líderes. }\end{array}$ & $\begin{array}{l}\text { "El microcrédito } \\
\text { a madres solteras } \\
\text { impacta a las } \\
\text { comunidades } \\
\text { indígenas" }\end{array}$ & $\begin{array}{l}\text { Medición de } \\
\text { percepciones a partir de } \\
\text { frecuencias que van de } \\
\text { "nunca" hasta "siempre" }\end{array}$ & Ordinal & $\begin{array}{l}\frac{\infty}{ \pm} \\
\underline{0} \\
\frac{0}{ \pm} \\
\pm\end{array}$ \\
\hline 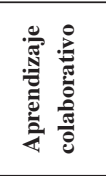 & $\begin{array}{l}\text { Refiere a percepciones de } \\
\text { prácticas profesionales } \\
\text { compartidas que anteriormente } \\
\text { se llevaban a cabo } \\
\text { discrecionalmente. }\end{array}$ & $\begin{array}{l}\text { “Antes podía visitar } \\
\text { a más comunidades } \\
\text { porque no tenía que } \\
\text { checar tarjeta” }\end{array}$ & $\begin{array}{l}\text { Medición de } \\
\text { percepciones a partir de } \\
\text { frecuencias que van de } \\
\text { "nunca" hasta "siempre" }\end{array}$ & Ordinal & $\begin{array}{l}\tilde{\sim} \\
\tilde{N} \\
\tilde{\Omega}\end{array}$ \\
\hline 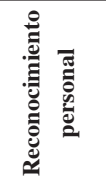 & $\begin{array}{l}\text { Son percepciones sobre la propia } \\
\text { práctica profesional en referencia } \\
\text { a los comentarios de líderes. }\end{array}$ & $\begin{array}{l}\text { "Me gusta mi labor } \\
\text { cuando entrego } \\
\text { despensas a la gente" }\end{array}$ & $\begin{array}{l}\text { Medición de } \\
\text { percepciones a partir de } \\
\text { frecuencias que van de } \\
\text { "nunca" hasta "siempre" }\end{array}$ & Ordinal & 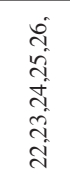 \\
\hline 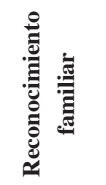 & $\begin{array}{l}\text { Son percepciones relativas a } \\
\text { logros que al ser resaltados por } \\
\text { familiares orientan la práctica } \\
\text { profesional hacia un orden } \\
\text { emocional. }\end{array}$ & $\begin{array}{l}\text { "Mi familia me } \\
\text { dice que le ayudo a } \\
\text { la gente que se ve } \\
\text { amolada" }\end{array}$ & $\begin{array}{l}\text { Medición de } \\
\text { percepciones a partir de } \\
\text { frecuencias que van de } \\
\text { "nunca" hasta "siempre" }\end{array}$ & Ordinal & $\begin{array}{l}\text { ते } \\
\infty \\
\text { Ṅ } \\
\text { ते }\end{array}$ \\
\hline 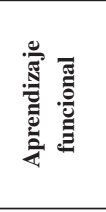 & $\begin{array}{l}\text { Son percepciones sobre retos } \\
\text { y oportunidades en referencia } \\
\text { a la práctica profesional que } \\
\text { los líderes de la institución han } \\
\text { implementado como normas } \\
\text { internas. } \\
\end{array}$ & $\begin{array}{l}\text { "Nuestro jefe nos } \\
\text { dice que debemos } \\
\text { involucrarnos con } \\
\text { las comunidades } \\
\text { indígenas" }\end{array}$ & $\begin{array}{l}\text { Medición de } \\
\text { percepciones a partir de } \\
\text { frecuencias que van de } \\
\text { "nunca" hasta "siempre" }\end{array}$ & Ordinal & $\frac{\pi}{2}$ \\
\hline 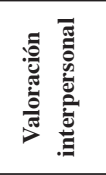 & $\begin{array}{l}\text { Refiere a percepciones alusivas } \\
\text { a la relevancia social de la } \\
\text { práctica profesional en relación } \\
\text { con comentarios de compañeros, } \\
\text { amistades o pareja. }\end{array}$ & $\begin{array}{l}\text { "Mis compañeros } \\
\text { están dispuestos a ir } \\
\text { a lugares de extrema } \\
\text { pobreza" }\end{array}$ & $\begin{array}{l}\text { Medición de } \\
\text { percepciones a partir de } \\
\text { frecuencias que van de } \\
\text { "nunca" hasta "siempre" }\end{array}$ & Ordinal & $\begin{array}{l}\stackrel{m}{2} \\
\tilde{m}\end{array}$ \\
\hline
\end{tabular}


El procedimiento de recopilación de información fue el siguiente: los encuestados expresan su grado de compromiso con respecto a situaciones específicas de sus funciones laborales y su entorno organizacional. A través de un contacto telefónico con la muestra seleccionada en la que se les solicitó una entrevista y cuyos fines serían meramente académicos e institucionales de seguimiento a los egresados, fuesen o no titulados. Una vez establecida la cita, se procedió a facilitarles un cuestionario donde se incluyeron las preguntas sociodemográficas, económicas y psicológicas organizacionales. En los casos en los que había una tendencia a la misma opción de respuesta, o bien la ausencia de respuesta, se les solicitó que anotaran al reverso las razones por las que contestaron con la misma opción de respuesta o, en su caso, la ausencia de ellas. Los datos fueron capturados en el Programa Estadístico para Ciencias Sociales (SPSS, por sus siglas en inglés) y los análisis de ecuaciones estructurales se estimaron con la ayuda del programa de Análisis de Momentos Estructurales (AMOS, por sus siglas en inglés) y el programa de Relaciones Estructurales Lineales (LISREL, por sus siglas en inglés).

El establecimiento del modelo estructural de relaciones reflejantes entre el compromiso laboral y sus indicadores se llevó a cabo considerando la normalidad, confiabilidad y validez de la escala que midió el constructo psicológico. El cuadro 3 muestra los resultados de los análisis de dicha normalidad, confiabilidad y validez.

El parámetro de curtosis fue empleado para establecer la normalidad de la distribución de respuestas ante el nivel de compromiso cuestionado. Los resultados muestran que el parámetro de curtosis tuvo un valor inferior a ocho que es el mínimo sugerido para asumir la normalidad de distribución. En el caso de la confiabilidad, el valor alfa de Cronbach permitió establecer la relación entre cada pregunta y la escala. El valor superior a 0.60 fue considerado como evidencia de una consistencia interna. Por último, el análisis factorial de componentes principales y rotación varimax en el que los pesos factoriales superiores a 0.300 permitieron deducir la emergencia del compromiso a partir de ocho indicadores. 


\section{Cuadro 3}

Distribución normal, análisis factorial y confiabilidad del compromiso organizacional

\begin{tabular}{l|c|c|c|c|c}
\hline \multicolumn{1}{c|}{ Factor } & Media & Desviación & Sesgo & Curtosis & Peso factorial \\
\hline Institucional & 3.1 & 0.24 & -1.378 & 0.985 & 0.304 \\
Supraordinal & 2.1 & 0.12 & -1.194 & 0.548 & -0.385 \\
Asistencial & 2.5 & 0.25 & 1.878 & 2.981 & 0.465 \\
Colaborativo & 2.6 & 0.13 & 1.280 & 0.446 & 0.305 \\
Personal & 2.0 & 0.15 & 1.362 & 0.887 & -0.567 \\
Familiar & 1.7 & 0.17 & 2.152 & 3.761 & 0.687 \\
Funcional & 3.5 & 0.10 & 2.043 & 3.746 & -0.342 \\
Interpersonal & 4.6 & 0.34 & -0.885 & -0.333 & 0.723 \\
\hline
\end{tabular}

Multivariate $=7.272 ;$ Bootstrap $=0000 ;$ Alfa $=0.673 ; \mathrm{KMO}=0.875 ; \mathrm{X}^{2}=12.156 ; 11 \mathrm{gl} ; \mathrm{p}=0.000$

Una vez establecida la normalidad, confiabilidad y validez, se procedió a establecer las covarianzas entre los indicadores para modelar las relaciones existentes con el factor organizacional.

\section{Resultados}

El análisis de covarianzas muestra asociaciones negativas y positivas, significantes y espurias entre los indicadores del compromiso laboral (véase cuadro 4). En el caso del compromiso que la muestra encuestada tiene con la institución en la que labora está relacionado con el compromiso de crecimiento en pareja $(\Phi=0.901)$; es decir, a medida que los objetivos institucionales se cumplen, éstos parecen incidir en los objetivos compartidos con alguna pareja. En este sentido, la dinámica interpersonal podría estar interrelacionada con otras dinámicas colaborativas que en el ámbito laboral son inherentes al clima de tareas o al clima de relaciones (Medina, Munduate, Martínez, Dorado y Mañas, 2004).

En contraste, el compromiso consigo mismo, que refleja una propensión al individualismo en referencia al compromiso institucional, el cual implica una propensión al colectivismo, es un indicador opuesto, ya que mientras los valores de una variable se incrementan se observa una disminución en los valores de la otra variable $(\Phi=-0.635)$. Esto es que los propósitos personales parecen no converger con los intereses de la institución en donde los profesionales del trabajo social ejercen sus funciones. 
En el caso del indicador supraordinal, el cual denota un compromiso más allá de las simples funciones de observación, entrevista, sistematización e intervención que la profesión exige, la crítica de estas funciones está vinculada con los objetivos interpersonales $(\Phi=0.650)$. En tal sentido, el clima de tareas y el clima de relaciones están más próximos al compromiso crítico de la profesión que al conflicto, ya que el cuestionamiento de las funciones asistencialistas es menester en el desarrollo del trabajo social (Mojoyinola, 2006). Quizá es por esta razón que la crítica de la profesión mantuvo una relación negativa con el compromiso asistencial $(\Phi$ $=-0.434)$.

\section{Cuadro 4}

\section{Covarianzas entre los determinantes del compromiso organizacional}

\begin{tabular}{l|c|c|c|c|c|c|c|c}
\hline & Institucional & Supraordinal & Asistencial & Colaborativo & Personal & Familiar & Funcional & Interpersonal \\
\hline Institucional & 0.072 & & & & & & & \\
Supraordinal & 0.710 & 0.594 & & & & & & \\
Asistencial & $\mathbf{- 0 . 5 8 6}$ & $\mathbf{- 0 . 4 3 4}$ & 0.042 & & & & & \\
Colaborativo & $\mathbf{- 0 . 3 8 8}$ & -0.038 & 0.035 & 0.210 & & & & \\
Personal & $\mathbf{- 0 . 6 3 5}$ & -0.009 & 0.187 & 0.055 & 0.103 & & & \\
Familiar & -0.188 & $\mathbf{- 0 . 3 7 5}$ & $\mathbf{0 . 6 2 8}$ & $\mathbf{0 . 7 2 5}$ & $\mathbf{0 . 6 5 2}$ & 0.677 & & \\
Funcional & -0.224 & -0.230 & $\mathbf{0 . 3 8 8}$ & 0.198 & $\mathbf{0 . 7 0 4}$ & 0.205 & 0.195 & \\
Interpersonal & 0.901 & 0.650 & -0.160 & -0.200 & $\mathbf{- 0 . 7 7 6}$ & $\mathbf{- 0 . 5 2 8}$ & 0.170 & 0.592 \\
\hline
\end{tabular}

Por su parte, el compromiso asistencial tuvo su mayor vinculación con el compromiso familiar $(\Phi=0.628)$. Ambos indicadores mantuvieron relaciones positivas a partir de las cuales es posible deducir que la muestra encuestada manifiesta una relación estrecha entre el ejercicio de la profesión como un compromiso asistencial y el tipo de grupo al que pertenecen o quieren pertenecer. Se trata de una tercera socialización del profesional del trabajo social en el que las políticas públicas y los programas asistencialistas parecen complementar la socialización de segundo orden de las familias y los grupos que rodearon a los profesionales del trabajo social en su desarrollo.

Por el contrario, el compromiso asistencialista está relacionado negativamente con el compromiso interpersonal $(\Phi=-0.160)$. A pesar de que la relación es espuria, otras variables ejercen influencia sobre ambos compromisos, tal asociación es relevante ya que el compromiso con la institución que proyecta y dirige los programas sociales se desvanece ante la interrelación del profesional del trabajo social con otros colegas, grupos vulnerables, referencia o pertenencia. El compromiso con el círculo interpersonal más cercano es opuesto a la política institucional 
En el caso de la relación entre el compromiso colaborativo y el compromiso familiar $(\Phi=0.725)$ es posible observar que el grupo de socialización primaria funge como un complemento del grupo de socialización de segundo orden. En términos sistémicos, el mesosistema en el que la familia y el trabajo son indicadores por excelencia puede ser explicado a partir del compromiso como un factor multidimensional (Bronfenbrenner, 1977 y 1994). No obstante, el compromiso colaborativo está relacionado negativamente con el compromiso interpersonal $(\Phi=-0.200)$.

Por otra parte, el compromiso consigo mismo está asociado positivamente con el compromiso hacia la profesión: el trabajo social $(\Phi=0.704)$. Las identidades personal y laboral al estar vinculadas explican el ejercicio consistente de la profesión, aun a pesar de las vicisitudes de la misma. En un contexto en el que las funciones del trabajo social se reducen a procedimientos técnicos y riesgo inherentes a los estudios socioeconómicos, los profesionales del trabajo social manifiestan una asociación estrecha entre las funciones de su trabajo y los objetivos personales de vida, pero tales aspectos son opacados por las relaciones con sus compañeros de profesión, ya que el compromiso con la profesión es opuesto al compromiso interpersonal $(\Phi=-0.776)$.

Cabe resaltar que la asociación entre el compromiso institucional y el supraordinal $(\Phi=0.710)$ supone que las normas de los centros de salud y el reconocimiento de los jefes hacia la práctica profesional de las trabajadoras sociales están asociados de un modo tal que el otorgamiento de recursos, los estudios socioeconómicos o las visitas domiciliarias parecen estar vinculadas a la estructura organizacional en cuanto a la distribución de los recursos o microcréditos.

En el caso del compromiso personal y aquel que se deriva del reconocimiento familiar $(\Phi=0.652)$ sugieren una interlocución entre la información que los familiares tienes del trabajo social y la práctica profesional de la misma. Este hallazgo es relevante a la luz del modelo de Caykoylu et al. (2011), ya que confirma la hipótesis relativa a que el compromiso es el resultado de factores organizacionales en referencia a los deseos personales. 


\section{Figura 4}

\section{Determinantes sociodemográficos del compromiso organizacional}

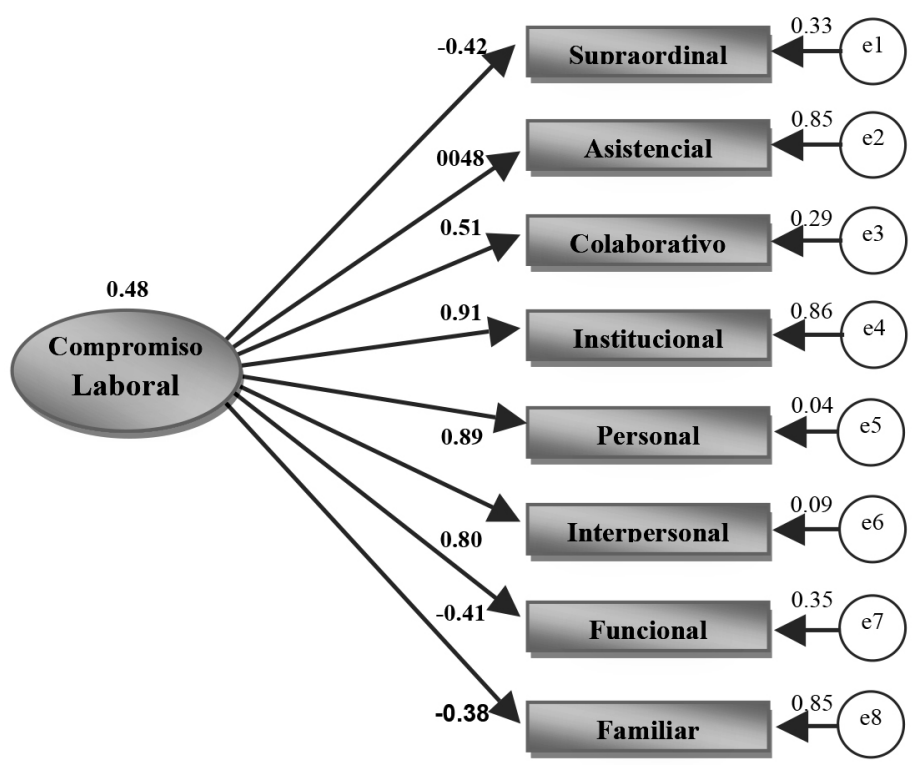

Una vez establecidas las covarianzas entre los indicadores, se procedió a estimar un modelo reflejante. La figura 4 muestra una mayor correlación entre el compromiso laboral-organizacional y el indicador institucional $(\beta=0.91)$. En este sentido, el constructo psicológico del compromiso laboral-organizacional es explicado por el compromiso hacia la política institucional. Al parecer los profesionales del trabajo social están influidos por los principios organizacionales más que por los objetivos personales, interpersonales, colaborativos, funcionales, profesionales, asistenciales, familiares o críticos inherentes al trabajo social.

En un sentido opuesto, el compromiso crítico de la profesión se relacionó negativamente con el factor $(\beta=-0.42)$. Tal resultado complementa el supuesto en torno al cual los profesionales del trabajo social ajustan sus objetivos a las políticas internas de la institución para la cual laboran, aunque coexisten con otros principios que guían el compromiso organizacional. 
Por último, se estimaron los índices de ajuste y residuo para contrastar la hipótesis relativa a la configuración de un compromiso organizacional que tendría como indicadores aspectos inherentes al individuo, familia, colegas, funciones, políticas y estructura en la que cada trabajador social está inserto (véase cuadro 5).

\section{Cuadro 5}

\section{Índices de ajuste de la estructura del compromiso organizacional}

\begin{tabular}{c|c|c|c|c|c|c}
\hline $\begin{array}{c}\text { Chi } \\
\text { Cuadrada }\end{array}$ & $\begin{array}{c}\text { Grados de } \\
\text { libertad }\end{array}$ & $\begin{array}{c}\text { Nivel de } \\
\text { significancia }\end{array}$ & $\begin{array}{c}\text { Índice de } \\
\text { Bondad de } \\
\text { Ajuste (GFI) }\end{array}$ & $\begin{array}{c}\text { Índice de } \\
\text { Ajuste } \\
\text { Normal (NFI) }\end{array}$ & $\begin{array}{c}\text { Índice de Ajuste } \\
\text { Comparado (CFI) }\end{array}$ & $\begin{array}{c}\text { Error de } \\
\text { Aproximación } \\
\text { Cuadrático Medio } \\
\text { (RMSEA) }\end{array}$ \\
\hline 17.00 & 0.13 & 0.000 & 0.975 & 0.975 & 0.985 & 0.009 \\
\hline
\end{tabular}

Los resultados muestran que la hipótesis nula puede ser aceptada, pues los índices de ajuste son próximos a la unidad y el residual cercano al cero.

\section{Conclusiones}

El presente estudio ha establecido ocho dimensiones del compromiso organizacional para evidenciar las diferencias entre el compromiso derivado de una socialización primaria que se observó en los objetivos personales, interpersonales, familiares y colaborativos en comparación al compromiso derivado de una socialización de segundo orden que se observó en los principios asistenciales, institucionales funcionales y supraordinales.

La convergencia entre estos principios de localización contextual e identidad ante relaciones de poder permiten deducir que el compromiso laboral es un entramado de percepciones, creencias, actitudes, decisiones y acciones encaminadas a la interrelación entre las ocho dimensiones esgrimidas.

Sin embargo, la prevalencia del compromiso institucional parece mostrar que los profesionales del trabajo social ajustan sus objetivos a la estructura y política de la organización para la cual trabajan.

Tales hallazgos son relevantes a la luz de la Teoría del Compromiso Laboral, ya que complementan uno de sus principios relativo a la atención al cliente. En la medida en que las organizaciones siguen una política de evaluación y satisfacción de la calidad del servicio propician un incremento en las responsabilidades, expec- 
tativas y compromisos de sus empleados (Caykoylu et al., 2011). De este modo, el presente estudio ha encontrado que el ajuste a las normas y políticas de una organización prevalece sobre los objetivos personales, interpersonales, colaborativos y familiares; es decir, si en las instituciones donde laboran los profesionales del trabajo social se siguieran políticas de calidad del servicio y a partir de evaluaciones consecutivas se estableciera la productividad, la muestra encuestada ajustaría sus compromisos primarios a los objetivos de dicha institución.

Sin embargo, la estructura de mando, de acuerdo con los estudios de Anwar y Norulkamar (2012) y de Tayo y Adeyemi (2012), muestra que el estilo de liderazgo explica un mayor porcentaje de la varianza del compromiso laboral en referencia con la satisfacción de vida, el desempeño o la productividad. En este sentido, este trabajo sostiene que el compromiso hacia la institución - al coexistir con el compromiso personal - explica la influencia del estilo de liderazgo. En el contexto del estudio, la muestra encuestada manifestó un compromiso laboral consistente en adecuar sus expectativas con la misión y visión de la institución donde laboran. Por ello, es lógico pensar que el estilo de liderazgo complementa la socialización primaria.

A pesar de las contribuciones esgrimidas, se recomienda extender el estudio a los estilos de liderazgo para desarrollar una teoría que permita explicar la influencia de la estructura de poder institucional en el compromiso laboral de los profesionales del trabajo social. Si se considera que en las instituciones los liderazgos son ocupados por otros profesionales de la salud, tales como administradores, contadores o médicos, entonces sería pertinente explicar las áreas de oportunidad para los profesionales del trabajo social al momento de asumir un compromiso y responsabilidad mayor: la planificación de una institución.

Ahora bien, con respecto a la construcción de un modelo explicativo de la satisfacción laboral como indicador de eficiencia en la práctica profesional del trabajo social, es menester considerar la incidencia de los programas sociales y estrategias sectoriales, ya que la dinámica de evaluación de políticas sociales como institucionales supone la obtención de objetivos por parte de un capital humano dispuesto a reproducir los planes de desarrollo sobre las necesidades sociales, así como gestionar las oportunidades y capacidades para difundir responsabilidades, tales como el de la salud en sectores vulnerables, marginados o excluidos. El éxito de las políticas de desarrollo está centrado en el nivel de compromiso de quienes llevan a cabo los planes y las estrategias institucionales, gestionan los recursos y promueven una cultura de autocuidado sin los cuales cualquier plan de desarrollo sería falible. 


\section{Referencias}

Anwar, F. y U. Norulkamar (2012). Mediating role of organizational commitment among leaderschip and employee outcomes, and empirical evidence from telecom sector. Processing International Seminar on Industrial Engineering and Management 2: 116-161.

Barranco, C., M. Delgado, C. Melin y R. Quintana (2010). Trabajo social en vivienda: investigación sobre calidad de vida percibida. Portularia 10: 101-111.

Bronfenbrenner, U. (1977). Toward an experimental ecology of human development. American Psychologist 32: 523-530.

(1994). Ecological models of human development. En M. Gauvain (ed.). Reading of the development of children. Oxford: Elseiver: 37-43.

Castro, A. (2006). Teorías implícitas del liderazgo, contexto y capacidad de conducción. Anales de Psicología 22: 87-97.

Castro, M. y M. Martins (2010). The relationships between organizational climate and employee satisfaction in information and technology organization. Tydskriff vir Bredyfsielkunde 36: 1-9.

Caykoylu, S., C. Egri, S. Havlovic y C. Bradley (2011). Key organizational commitment antecedents for nurses, paramedical professionals and non-clinical staff. Journal of Health Organization and Management 25: 7-33.

Danes, S. y J. Juyoung (2013). Copreneural identity development during new venture creation. Journal of Family Business Management 3: 45-61.

Figeiredo, H., E. Grau, P. Gil y J. García (2012). Síndrome de quemarse por el trabajo y satisfacción laboral en profesionales de enfermería. Psocthema 24 : 271-276.

García-Lirios, C. (2011). Estructura del estrés laboral. Alternativas 25: 22-33.

Hermosa, A. (2006). Satisfacción laboral y síndrome de Bournout en profesores de educación primaria y secundaria. Revista Colombiana de Psicología 15: 81-89. 
Mañas, M., J. Boada, E. González, y E. Agullo (2007). La satisfacción y el bienestar psicológico como antecedentes del compromiso organizacional. Psicothema 19: 395-400.

Medina, F., L. Munduate, I. Martínez, M. Dorado y M. Mañas (2004). Efectos positivos de la activación del conflicto de tarea sobre el clima de los equipos de trabajo. Revista de Psicología Social 10: 3-15.

Melano, M. (2007). Ciudadanía y autonomía en trabajo social: el papel de los saberes políticos y científicos técnicos. Revista Alternativas 15: 99-110.

Mendoza, M., A. Orgambídez y A. Carrasco (2010). Orientación de la calidad total, satisfacción laboral, comunicación y compromiso en establecimientos de turismo rural. Revista de Turismo y Patrimonio Cultural 8: 351-361.

Mojoyinola, J. (2006). Social work interventions in the prevention and management in violence domestic. Journal of Social Science 13: 97-99.

Moreno, M., L. Ríos, J. Canto, J. García y F. Perles (2010). Satisfacción laboral y Burnout en trabajos poco cualificados: diferencias entre sexos en población inmigrante. Revista de Psicología del Trabajo y las Organizaciones 26: $255-265$.

Neal, A., M. Griffin y P. Hart (2000). The impact of organizational climate on safety climate and individual behavior. Safety Science 34: 99-109.

Ocampo, J. (2008). Las concepciones de la política social: universalismo versus focalización. Nueva Sociedad 215: 36-61.

Pastor, E. (2012). Sostenibilidad, impacto y eficacia de las políticas sociales municipales mediante la democratización e implicación social. Sociedad y estado 27: 563-588.

Ríos, M., M. Téllez y J. Ferrer (2010). El empoderamiento como predictor del compromiso organizacional en las Pymes. Contaduría y Administración 231: 103-125. 
Ruiz, L. (2010). La gestión urbana de la seguridad: política criminal y municipios. Revista de Ciencia Penal y Criminología 12: 1-125.

Salgado, J., G. Remeseiro y M. Iglesias (1996). Clima organizacional y satisfacción laboral en una Pyme. Psicothema 8: 329-335.

Santarsierro, L. (2012). Las políticas sociales en el caso de la satisfacción de necesidades. Algunos elementos conceptuales para su determinación. Trabajo y Sociedad 18: 159-176.

Tayo, E. y A. Adeyemi (2012). Job involvement \& organizational commitment as determinants of job performance among educational resource centre personal. European Journal of Globalization and Development Research 5: 301313.

Tejero, C. y M. Fernández (2009). Medición de la satisfacción laboral en la dirección escolar. Revista Electrónica de Investigación y Evaluación Educativa 15: 1-16.

Vecina, M., F. Chacón y N. Sueiro (2009). Satisfacción en el voluntariado: estructura interna y relación con la permanencia en las organizaciones. Psicothema 21: 112-117.

Wallece, J., J. Hunt y C. Richards. (1999). The relationships between organizational culture organizational climate and managerial values. International Public Sector Managerial 12: 548-564.

Yáñez, R., M. Arenas y M. Ripoll (2010). El impacto de las relaciones interpersonales en la satisfacción laboral. Liberabit 16: 193-202. 
